\title{
Measurements and modeling of coherent synchrotron radiation and its impact on the Linac Coherent Light Source electron beam
}

\author{
K. L. F. Bane, F.-J. Decker, Y. Ding, D. Dowell, P. Emma, J. Frisch, Z. Huang, ${ }^{*}$ R. Iverson, C. Limborg-Deprey, H. Loos, \\ H.-D. Nuhn, D. Ratner, G. Stupakov, J. Turner, J. Welch, and J. Wu \\ SLAC National Accelerator Laboratory, Menlo Park, California 94025, USA
}

(Received 3 December 2008; published 17 March 2009)

\begin{abstract}
In order to reach the high peak current required for an x-ray free electron laser, two separate magnetic dipole chicanes are used in the Linac Coherent Light Source accelerator to compress the electron bunch length in stages. In these bunch compressors, coherent synchrotron radiation (CSR) can be emitted either by a short electron bunch or by any longitudinal density modulation that may be on the bunch. In this paper, we report detailed measurements of the CSR-induced energy loss and transverse emittance growth in these compressors. Good agreement is found between the experimental results and multiparticle tracking studies. We also describe direct observations of CSR at optical wavelengths and compare with analytical models based on beam microbunching.
\end{abstract}

DOI: 10.1103/PhysRevSTAB.12.030704

\section{INTRODUCTION}

Coherent synchrotron radiation (CSR) is one of the most challenging issues associated with the design of bunch compressor chicanes for advanced accelerators and x-ray facilities. In recent years, effects of CSR on electron beam quality have been studied extensively in theory and simulations (see, e.g., Ref. [1] for a recent review). Experimental studies of CSR effects are relatively sparse because adequate beam quality is not commonly available. Examples of previous measurements can be found in Refs. [2-6]. In this paper, we present detailed measurements of CSR-induced energy loss and transverse emittance growth at two bunch compressors for the Linac Coherent Light Source (LCLS), an x-ray free-electron laser currently in the final phase of the commissioning at SLAC. The experimental results are compared with extensive tracking studies.

CSR can be emitted not only for wavelengths longer than the electron bunch length but also for shorter wavelengths if the electron beam has high-frequency density modulations caused by a microbunching instability $[7,8]$. It was found during the LCLS injector commissioning [9] that the presence of a microbunched beam contributes significantly to the enhancement of the optical signal on optical transition radiation (OTR) diagnostic screens after bends. In this paper, we also report direct observations of CSR at optical wavelengths on one of the LCLS OTR screens and compare with analytical models based on beam microbunching.

\section{EXPERIMENTAL BACKGROUND}

Figure 1 shows a schematic of the LCLS linacs and two bunch compressors ( $\mathrm{BC} 1$ and $\mathrm{BC} 2)$, with nominal beam

*zrh@slac.stanford.edu
PACS numbers: 29.27.Bd, 41.60.Ap, 41.60.Cr, 41.75.Ht

energy listed at selected locations. Both compressors are four-dipole chicanes and are motorized so that the central two dipoles can be horizontally translated to match the beam trajectory. The horizontal dispersion is corrected after the chicanes using a pair of tweaker quads in the chicanes (see, e.g., BC1 layout in Fig. 2). The injector through BC1 was successfully commissioned in 2007 [9], and some preliminary results of $\mathrm{BC} 1$ compression and emittance growth were reported in Ref. [10]. The commissioning of the entire accelerator including $\mathrm{BC} 2$ has just been concluded in 2008 [11]. A "laser heater" (shown in Fig. 1) to Landau damp the microbunching instability by increasing the uncorrelated energy spread in the beam [12] will not be installed until late 2008. A relatively low charge of $250 \mathrm{pC}$ is used to tune up the machine and to perform these CSR measurements, although higher charge levels of $1 \mathrm{nC}$ have also been explored. The nominal linac and bunch compressor parameters at $250 \mathrm{pC}$ charge are listed in Tables I and II, where rf phases are defined with respect to the crest (with negative phase values indicating the beam is in front of the crest). Under typical operating conditions, the (rms) bunch length is compressed from 750 to $100 \mu \mathrm{m}$ after $\mathrm{BC} 1$, and then to $8 \mu \mathrm{m}$ after $\mathrm{BC} 2$ to reach a final current of about $2.5 \mathrm{kA}$.

\section{BC1 RESULTS}

\section{A. Measurements}

As is shown in Table I, at $250 \mathrm{pC}$ charge (and $135 \mathrm{MeV}$ energy), the measured (rms) bunch length is typically $750 \mu \mathrm{m}$ and the normalized emittances (in $x$ and $y$ ) are $0.7 \mu \mathrm{m}$. The bunch length is measured using a transverse deflecting cavity located in the injector area [13] (TCAV0 in Fig. 1), and the emittances are measured using an OTR screen and a wire scanner before a $35^{\circ}$ achromatic bend (DL1). In order to study the CSR effects on BC1 compres- 


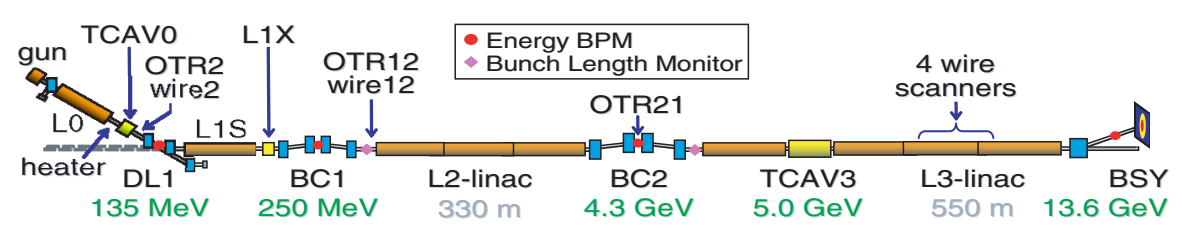

FIG. 1. (Color) LCLS accelerator layout showing BC1, BC2, both transverse rf cavities (TCAV0 and TCAV3), and screen at end of linac. The laser heater has not been installed for the experiments described in this paper.

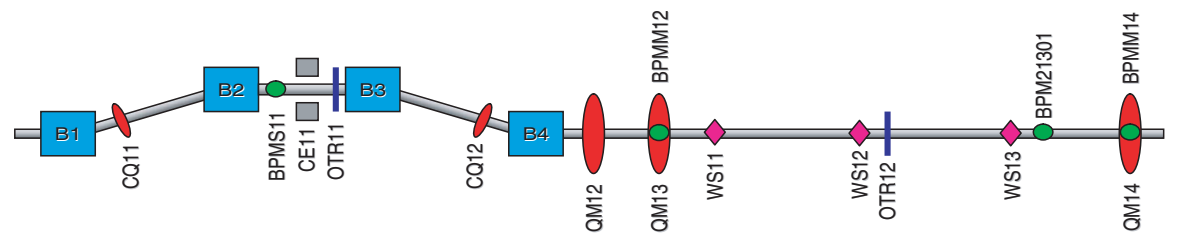

FIG. 2. (Color) Bunch compressor 1 (BC1) and its diagnostics section showing tweaker quads (CQ11 and CQ12) in the chicane for dispersion correction, BPMs, quadrupoles, OTR screens, and wire scanners (WS) for beam diagnostics.

sion, the incoming energy chirp of the electron bunch is varied by adjusting the L1S rf phase from $-20^{\circ}$ to $-30^{\circ}$ while holding the $\mathrm{BC} 1$ energy constant at $250 \mathrm{MeV}$ and the $\mathrm{BC} 1 R_{56}$ constant at $-45.5 \mathrm{~mm}$. The short $x$-band section (L1X), a 4th harmonic rf system used to linearize the longitudinal phase space (by decelerating the bunch by

TABLE I. Nominal measured beam and linac parameters for 250-pC bunch charge operation.

\begin{tabular}{lccc}
\hline \hline Parameter & Symbol & Value & Unit \\
\hline Injector bunch length (rms) & $\sigma_{z 0}$ & $\sim 750$ & $\mu \mathrm{m}$ \\
Injector normalized emittance $(x / y)$ & $\gamma \epsilon_{0}$ & $\sim 0.7$ & $\mu \mathrm{m}$ \\
L1S rf phase (2856 MHz) & $\psi_{1}$ & -22 & $\mathrm{deg}-\mathrm{S}$ \\
L1X rf phase (11.4 GHz) & $\psi_{X}$ & -160 & $\mathrm{deg}-\mathrm{X}$ \\
L1X rf peak voltage & $V_{X}$ & 20 & $\mathrm{MV}$ \\
L2 rf phase (2856 MHz) & $\psi_{2}$ & -37 & $\mathrm{deg}-\mathrm{S}$ \\
L3 rf phase (2856 MHz) & $\psi_{3}$ & 0 & $\mathrm{deg}-\mathrm{S}$ \\
Final electron energy & $E_{f}$ & 13.6 & $\mathrm{GeV}$ \\
Final bunch length (rms) & $\sigma_{z f}$ & $\sim 8$ & $\mu \mathrm{m}$ \\
Final normalized emittance $(x / y)$ & $\gamma \epsilon_{f}$ & $\sim 1.3 / 0.7$ & $\mu \mathrm{m}$ \\
\hline \hline
\end{tabular}

TABLE II. BC1 and BC2 chicane parameters at $250 \mathrm{pC}$.

\begin{tabular}{lcccc}
\hline \hline Parameter & Symbol & BC1 & BC2 & Unit \\
\hline Electron energy & $E_{0}$ & 0.25 & 4.3 & $\mathrm{GeV}$ \\
Energy spread (rms) & $\sigma_{E} / E_{0}$ & 1.4 & 0.38 & $\%$ \\
Momentum compaction & $R_{56}$ & -45.5 & -24.7 & $\mathrm{~mm}$ \\
Chicane total length & $L_{T}$ & 6.5 & 23 & $\mathrm{~m}$ \\
Bend angle per dipole & $|\theta|$ & 5.4 & 2.0 & $\mathrm{deg}$ \\
Effective length per dipole & $L_{B}$ & 0.20 & 0.54 & $\mathrm{~m}$ \\
Dipole bending radius & $\rho$ & 2.1 & 15.5 & $\mathrm{~m}$ \\
B1 to B2 ( = B3 to B4) & $\Delta L$ & 2.43 & 9.87 & $\mathrm{~m}$ \\
Dispersion at center & $|\eta|$ & 247 & 363 & $\mathrm{~mm}$ \\
Translation range & $\Delta x$ & $0-30$ & $0-52$ & $\mathrm{~cm}$ \\
\hline \hline
\end{tabular}

about $19 \mathrm{MV}$ ), is also held constant in phase and amplitude.

To measure the absolute bunch length after $\mathrm{BC} 1$, the BC2 chicane is switched off and the bunch is vertically streaked using a transverse deflecting cavity located at $5 \mathrm{GeV}$ (TCAV3 in Fig. 1). The phosphor screen located at the beam switchyard (BSY) after the L3 linac records the vertical beam size, which is then converted to bunch length due to the imposed $y-z$ correlation at TCAV3. The phosphor screen exhibits some persistence of beam images at higher bunch repetition rates. Because of vertical position jitters on the screen, the measured bunch lengths shown in Fig. 3 are usually reported longer at the $10-\mathrm{Hz}$ repetition rate than at $1 \mathrm{~Hz}$. Figure 3 also shows that the bunch is undercompressed above $-27^{\circ}$ and overcompressed below $-27^{\circ}$, with the minimum measured rms bunch length of $15 \mu \mathrm{m}$ at full compression. (The simulations shown here

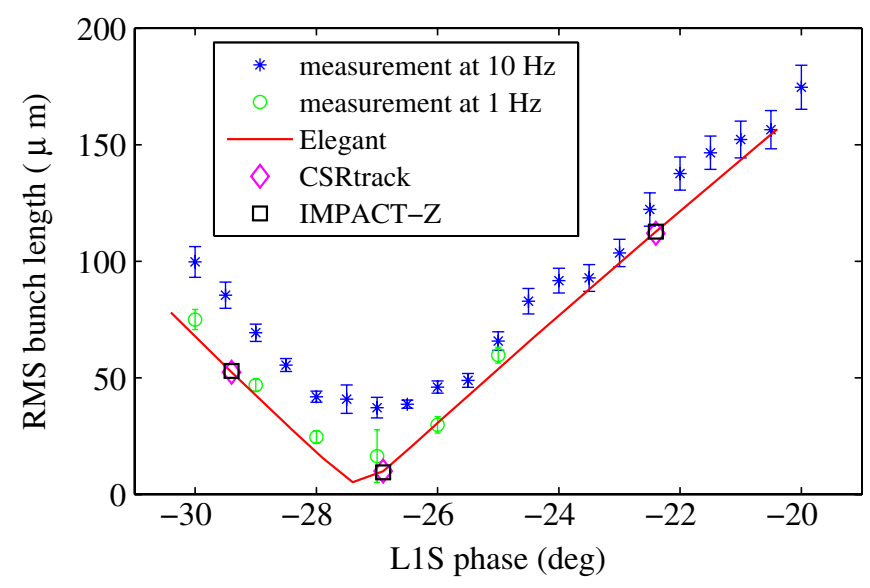

FIG. 3. (Color) BC1 compression measurements and simulations vs L1S phase (zero phase is on crest). The $1-\mathrm{Hz}$ (green) measurements are thought to be more accurate, due to a screen persistence problem at $10 \mathrm{~Hz}$ (blue). 
are taken from three separate computer codes, ELEGANT, CSRTRACK, and IMPACT-Z, to be described below in Sec. III B.)

In a dipole magnet, coherent radiation of a Gaussian bunch entails an average energy loss per electron per unit length of [14]

$$
\frac{d E}{d s} \approx \frac{1.8 N e^{2}}{\rho^{2 / 3} \sigma_{z}^{4 / 3}},
$$

where $N=1.56 \times 10^{9}$ is total number of electrons (at $250 \mathrm{pC}$ ), and $\rho=2.1 \mathrm{~m}$ is the $\mathrm{BC} 1$ dipole bending radius (see Table II). A compressed bunch with rms length $\sigma_{z}$ traversing the last dipole of $\mathrm{BC} 1$ (which is $0.2 \mathrm{~m}$ in length) can induce significant energy loss due to CSR (about $2 \mathrm{MeV}$ for $\sigma_{z}=10 \mu \mathrm{m}$ at $250 \mathrm{pC}$ ). Since the energy loss occurs primarily in the last dipole, the bunch will be kicked horizontally and will execute a betatron oscillation after the bend. A beam position monitor (BPM) after BC1 (BPMM12 in Fig. 2) shows the expected horizontal steering effect due to CSR, which can be utilized in a quick scan to identify the full compression phase (see Fig. 4).

Also associated with the CSR energy loss, the bunch energy spread can increase in the chicane, giving rise to horizontal emittance growth after the chicane. A quadrupole magnet 1.0 meters after $\mathrm{BC} 1$ (QM13) and a wire scanner 2.2 meters after the quadrupole (WS12 in Fig. 2) are used to measure the projected emittances in both $x$ and $y$ using the "quad-scan" technique. Two separate sets of horizontal emittance measurements and one set of vertical emittance measurements are shown in Figs. 5(a) and 5(b). In addition to the pronounced peak in horizontal emittance at full compression (at $-27^{\circ}$, as is shown in the simultaneous measurements of Fig. 3), a vertical emittance increase of $50 \%$ can also be seen. Note that $\mathrm{BC} 1$ normally

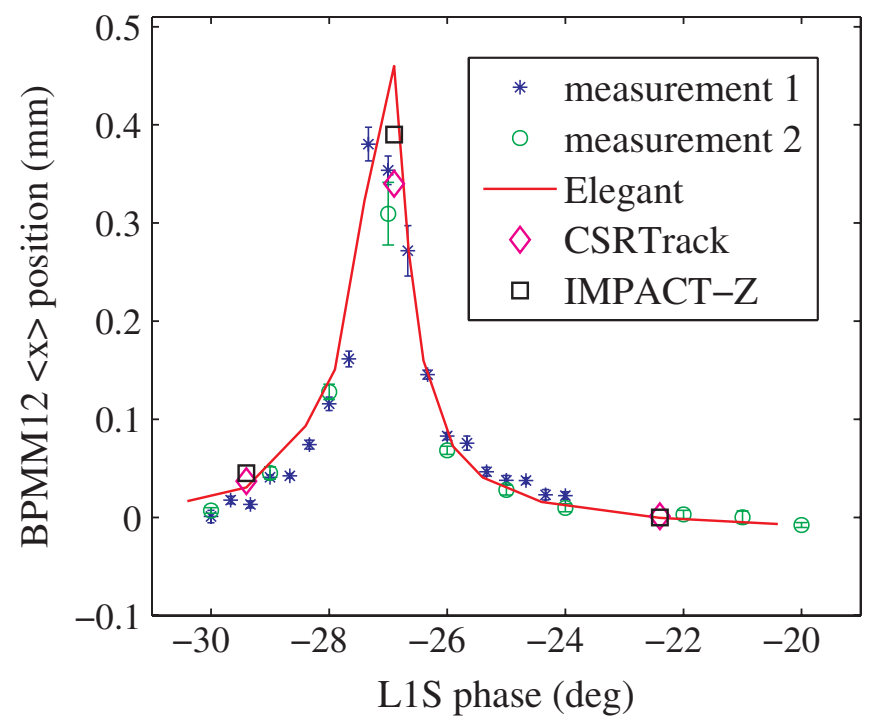

FIG. 4. (Color) Post-BC1 BPMM12 $x$ position vs L1S phase to monitor CSR energy loss.

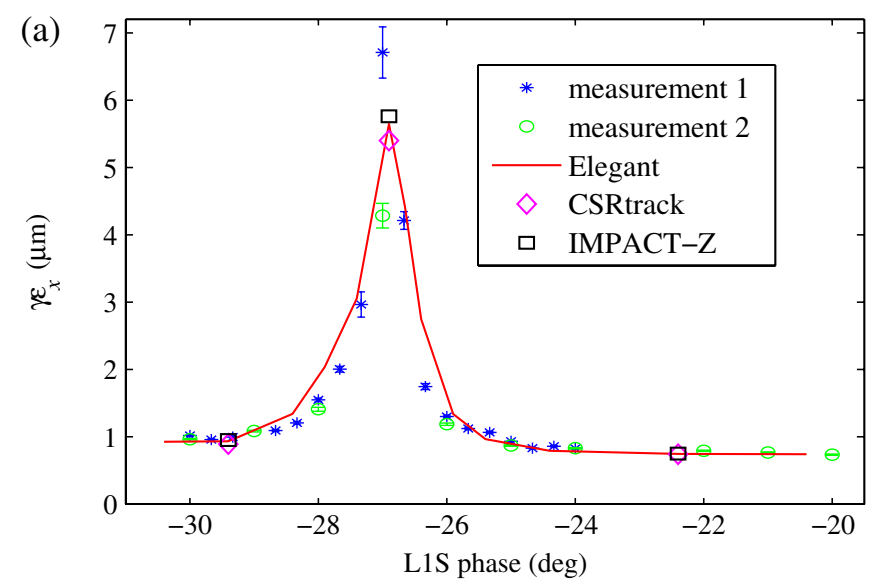

Horizontal emittance after BC1 at $250 \mathrm{pC}$.

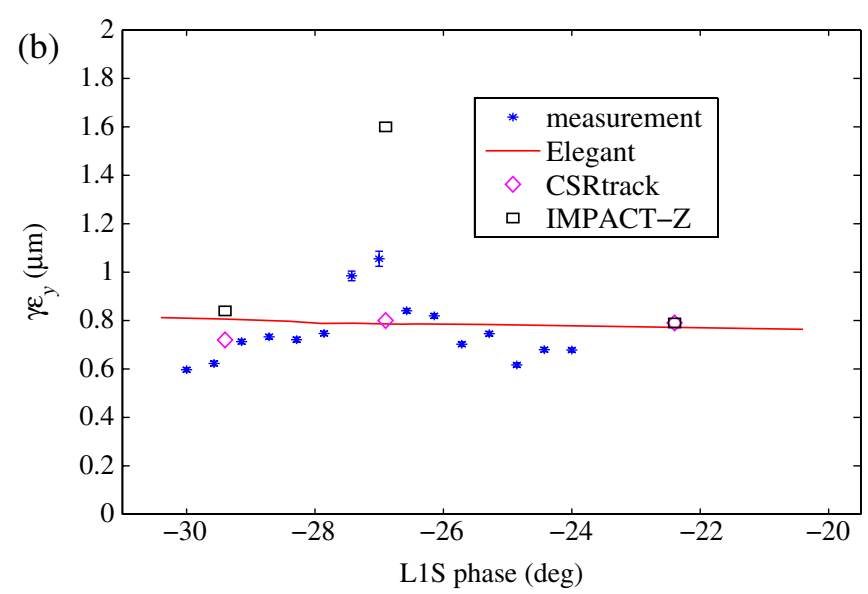

Vertical emittance after BC1 at $250 \mathrm{pC}$.

FIG. 5. (Color) Measured and simulated transverse emittances after BC1 vs L1S phase. (a) Horizontal emittance after BC1 at 250 pC. (b) Vertical emittance after BC1 at 250 pC.

operates at $-22^{\circ}$ (at a compression factor of $\sim 7$ ) where transverse emittances are unaffected by CSR. Note also that the emittances almost fully return to their uncompressed levels at phases below $-29^{\circ}$, i.e., when the bunch is overcompressed. Figures 6(a) and 6(b) show measured horizontal profiles of the beam at the wire scanner, for the nominal phase $\left(-22^{\circ}\right)$ and at full compression $\left(-27^{\circ}\right)$, respectively. The horizontal profile at full compression is much larger and very much distorted due to CSR and shotto-shot position jitter.

\section{B. Simulations}

Multiparticle simulations were used to compare with these measurements. First the injector (up to DL1 in Fig. 1) was modeled using the IMPACT-T [15] space charge code with one million macroparticles. The simulated normalized emittances and the rms bunch length at the injector end were chosen to replicate the measured values shown in Table II. These macroparticles were used as input for 

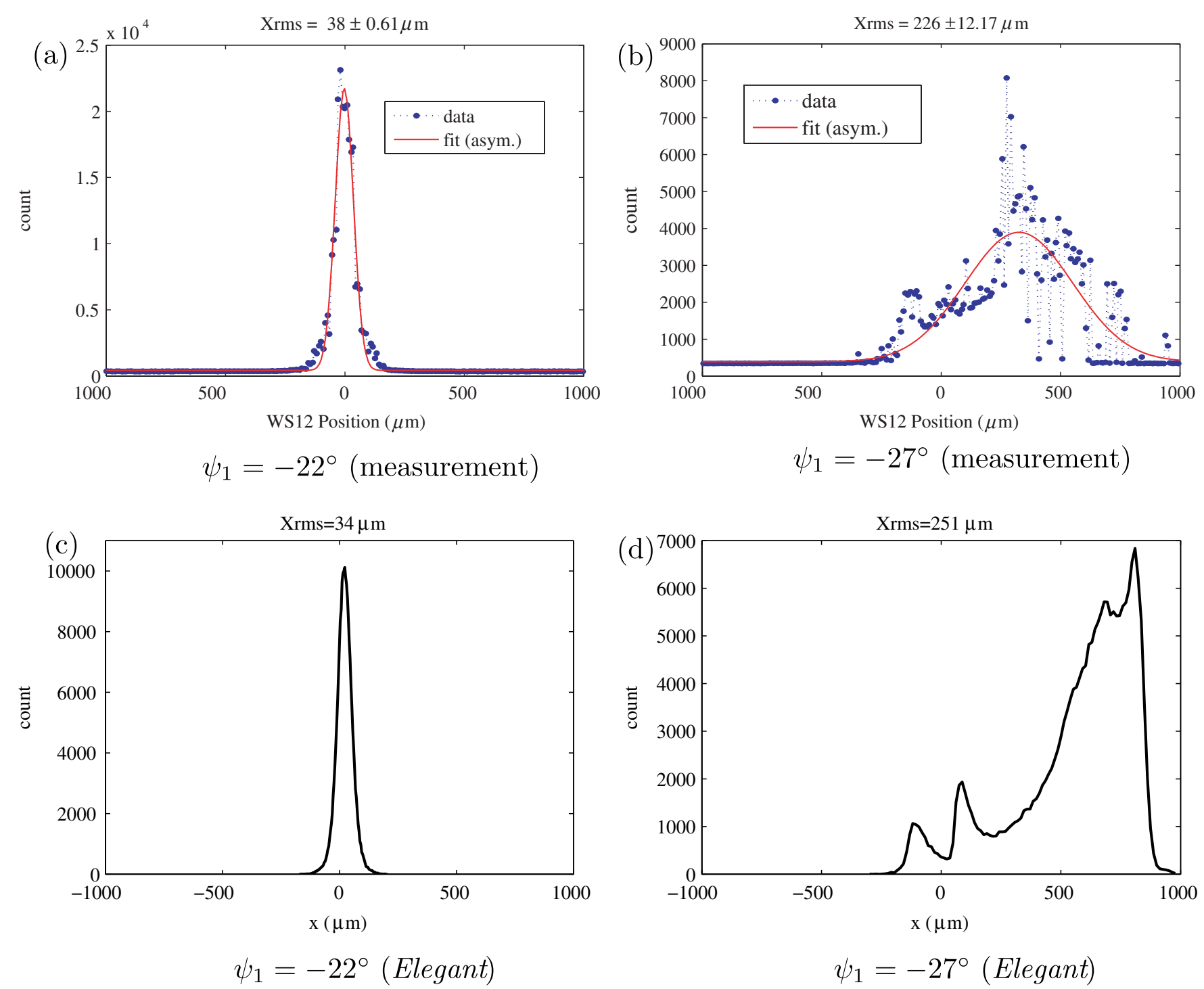

FIG. 6. (Color) Measured and simulated horizontal beam profiles at the post-BC1 wire scanner for two L1S phases. (a) $\psi_{1}=-22^{\circ}$ (measurement). (b) $\psi_{1}=-27^{\circ}$ (measurement). (c) $\psi_{1}=-22^{\circ}$ (ELEGANT). (d) $\psi_{1}=-27^{\circ}$ (ELEGANT).

ELEGANT [16] and for different L1S phases were tracked through $\mathrm{BC} 1$ with a 1D CSR model that includes field transients. A phase shift of $-0.4^{\circ}$ was added in the simulation results of Figs. 3-5, to fit with measurements of the compressed bunch length. (This phase shift may come from rf drift during the measurements or for a small uncertainty in the initial phase.) The BPMM12 horizontal position readout due to CSR energy loss is in good agreement with ELEGANT simulations (see Fig. 4). In addition, the simulated emittances are compared with the measurements in Figs. 5(a) and 5(b). The validity of the 1D CSR model was cross-checked using CSRTRACK [17] with a 2D $(x-z)$ CSR algorithm ("g_to_m" method) to track the beam in the $\mathrm{BC} 1$ region for three L1S phases as shown in Fig. 3 (when the beam is undercompressed, fully compressed, and overcompressed). CSRTRACK simu- lations yield the same rms bunch length (after applying the same $-0.4^{\circ}$ phase shift as in the ELEGANT case). Although the 2D CSRTRACK code predicts a somewhat different energy loss a few meters after the chicane, the energy loss up to the end of the last dipole is quite similar to that calculated by the 1D ELEGANT code. As a result, the BPMM12 readout of CSR energy loss, which only responds to the loss up to the end of the last chicane dipole, is very well modeled by both codes (Fig. 4). Good agreement is also found between the simulated horizontal emittances from both ELEGANT and CSRTRACK and the measurements. Figures 7(a)-7(f) show ELEGANT simulations of the longitudinal phase space distributions and the current profiles at WS12 for three L1S phases, resulting in a beam that is undercompressed, fully compressed, and overcompressed, respectively. 

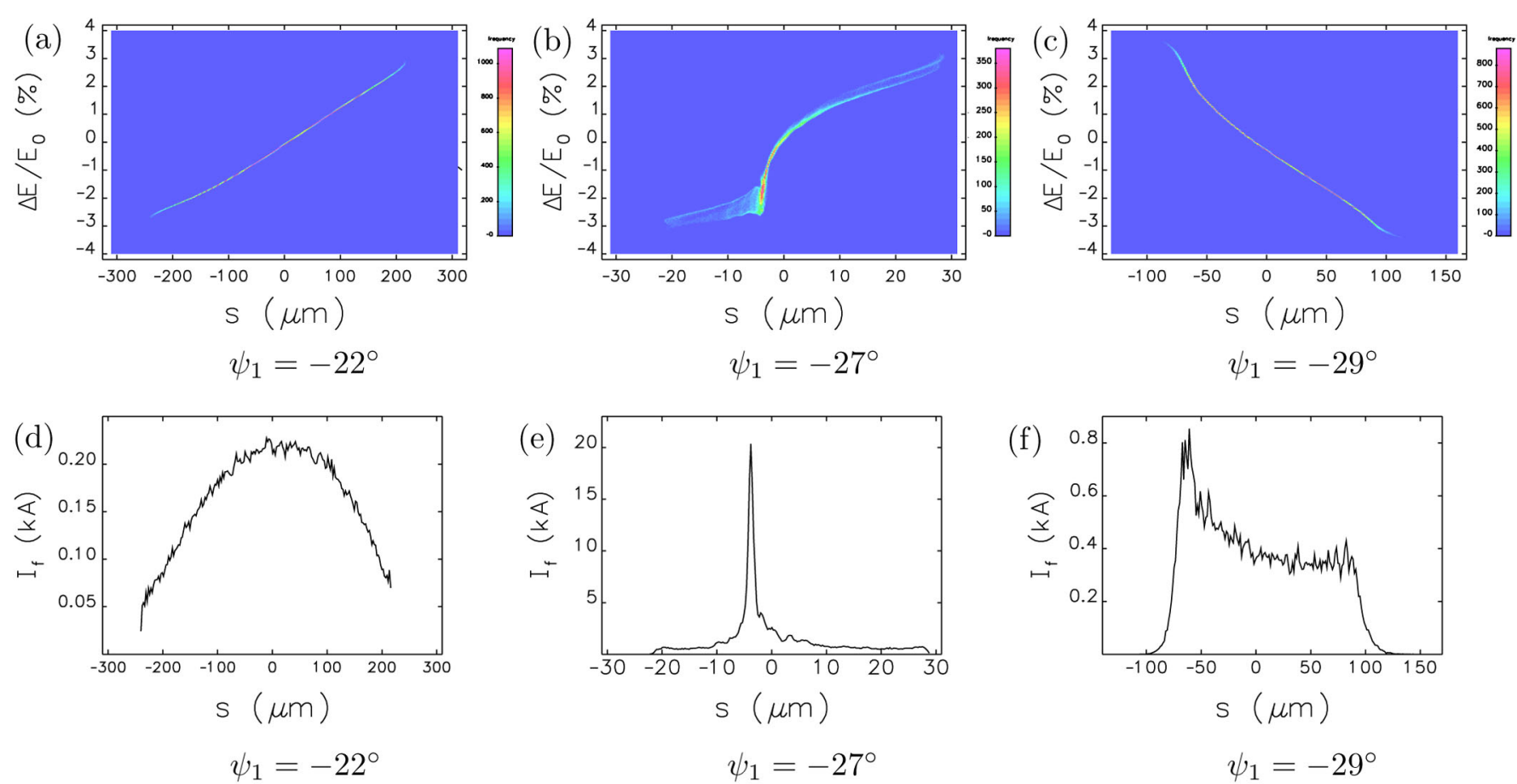

FIG. 7. (Color) ELEGANT simulations of the beam longitudinal phase space distributions (upper plots) and the current profiles (lower plots) after BC1 at $250 \mathrm{MeV}$ for three L1S phases. The bunch head is to the left. (a) $\psi_{1}=-22^{\circ}$. (b) $\psi_{1}=-27^{\circ}$. (c) $\psi_{1}=-29^{\circ}$. (d) $\psi_{1}=-22^{\circ}$. (e) $\psi_{1}=-27^{\circ}$. (f) $\psi_{1}=-29^{\circ}$.

Since no vertical self-force is present in these two codes, ELEGANT and CSRTRACK simulations do not show any vertical emittance growth, in contrast with the vertical measurement at full compression. However, these simulations indicate that a current spike of $\sim 20 \mathrm{kA}$ is formed after $\mathrm{BC} 1$ at the full compression phase [see Figs. 7(b) and 7(e)]. We suppose that the transverse space charge force at this high-current spike may increase the vertical emittance in the 3-m drift section after $\mathrm{BC} 1$ and before the beam reaches the wire scanner. Using a 3D space charge code IMPACT-Z that incorporates the same 1D CSR model as in ELEGANT [18], we simulate the three compression phases and plot the results in Figs. 4, 5(a), and 5(b). Figure 5(b) suggests that the increase in vertical emittance at full compression may be due to the vertical space charge effect after BC1. Finally, Figs. 6(c) and 6(d) give the simulated horizontal profiles as obtained by ELEGANT, to be compared with the measurements.

\section{BC2 RESULTS}

\section{A. Bunch length and emittance studies}

When the BC2 chicane is switched on, the L2-linac phase (see Fig. 1) can also be varied to measure the bunch compression and its effect on the beam. The results are less reliable than those of the $\mathrm{BC} 1$ studies because the emittance can be sensitive to the rf phase of the 330-m L2 linac section. In order to avoid this problem, we instead vary the strength of the $\mathrm{BC} 2$ chicane while holding the L2 linac phase fixed at $-37^{\circ}$, with $\mathrm{BC} 1$ in its nominal configuration as listed in Table II. The compressed bunch length is measured with the TCAV3 transverse rf deflector (see Fig. 1) at a $1-\mathrm{Hz}$ beam rate. The projected transverse emittances are measured with four wire scanners near the end of the linac (at about the $10 \mathrm{GeV}$ point, see Fig. 1). Figures 8(a) and 8(b) show the measured bunch length and horizontal emittance as functions of the $\mathrm{BC} 2$ chicane strength (again at $250 \mathrm{pC}$ charge). The rms bunch length measurements extend down to $\sim 2 \mu \mathrm{m}$ near full compression [see inset plot in Fig. 8(a)]. When BC2 is switched off, the horizontal emittance returns to the same level as the vertical emittance (not shown), both at the $0.7 \mu \mathrm{m}$ level under nominal $\mathrm{BC} 1$ compression. This confirms the emittance preservation through the linac in the absence of $\mathrm{BC} 2$ compression. However, when $\mathrm{BC} 2$ operates at the nominal strength $\left(R_{56}=-24.7 \mathrm{~mm}\right.$ at $\left.250 \mathrm{pC}\right)$, the horizontal projected emittance is almost doubled due to CSR. ELEGANT tracking of the entire accelerator including wakefields, longitudinal space charge in the linac, and CSR in $\mathrm{BC} 1$ and $\mathrm{BC} 2$ confirms the CSR emittance growth in $\mathrm{BC} 2$ as a function of compression [red curve in Fig. 8(b)]. The initial beam configurations are similar to the previous $\mathrm{BC} 1$ simulations, and $-1^{\circ}$ is added to the L2 phase in these simulations in order to match the bunch length measurements as shown in Fig. 8(a); again the discrepancies are likely due to small rf phase drifts during the experiment.

The longitudinal phase space of the compressed bunch is strongly affected by the longitudinal wakefield in the L2 


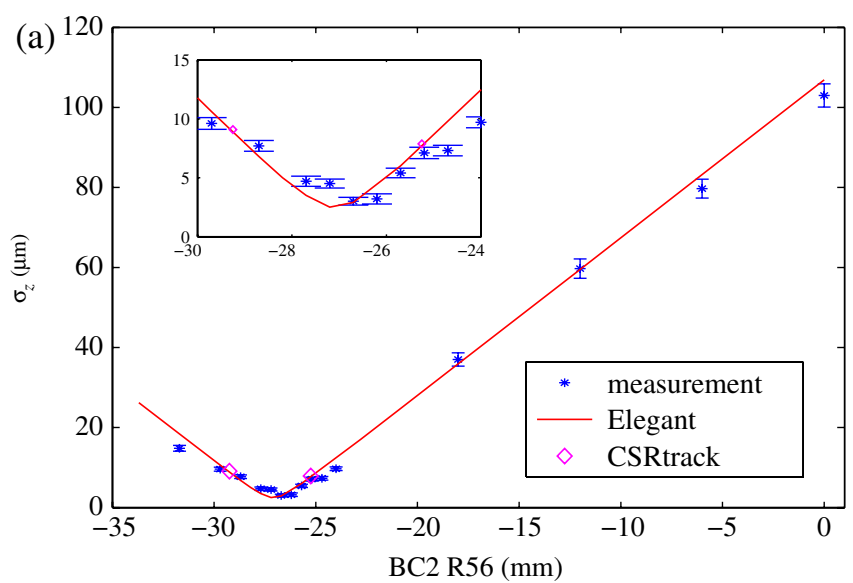

Compressed bunch length after BC2 at $250 \mathrm{pC}$.

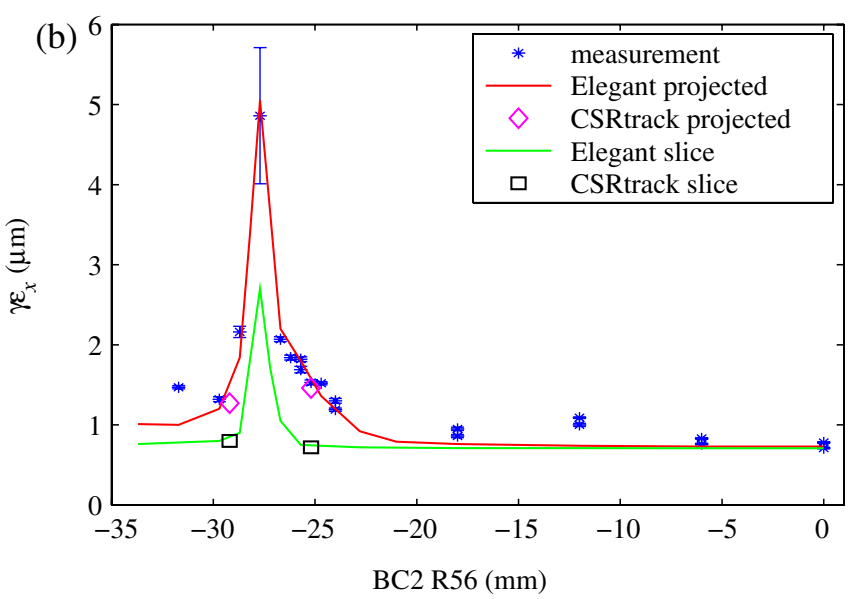

Horizontal emittance after BC2 at $250 \mathrm{pC}$.

FIG. 8. (Color) BC2 bunch length compression and emittance growth measurements and simulations. (a) Compressed bunch length after BC2 at 250 pC. (b) Horizontal emittance after BC2 at $250 \mathrm{pC}$.

linac, and the compressed bunch current profile is far from being a Gaussian or parabolic distribution. This is illustrated in ELEGANT simulations shown in Figs. 9(a)-9(f) for three $\mathrm{BC} 2$ strengths, corresponding to a beam that is undercompressed, slightly overcompressed, and significantly overcompressed, respectively. These plots correspond to the $10 \mathrm{GeV}$ point in the linac, where the projected emittances are measured by four wire scanners. The longitudinal phase space distributions may change after $\mathrm{BC} 2$ due to wakefields in the L3 linac but the current profiles remain the same as at the $\mathrm{BC} 2$ exit. When the bunch is undercompressed [see Figs. 9(a) and 9(d)], the simulated current profile has high-current leading and trailing spikes formed near the end of $\mathrm{BC} 2$. These high-current spikes contribute to most of the projected emittance growth. As ELEGANT simulations indicate, the slice emittance in the core part of the bunch is unaffected in this undercompressed regime [when $\left|R_{56}\right|<25 \mathrm{~mm}$, green curve in Fig. 8(b)]. The bunch from the injector simulation is heavily smoothed to remove as much numerical noise as possible before the ELEGANT runs are begun; nevertheless, the beam after BC2 is clearly microbunched at wavelengths much shorter than the bunch length due to the very high gain of the LCLS accelerator system at these short wavelengths [12] (see Fig. 9). As is shown in Figs. 9(b) and 9(e) the bunch longitudinal phase space becomes even more complicated near full compression. The simulated peak current reaches a maximum of more than $25 \mathrm{kA}$ when the bunch is slightly overcompressed (in terms of the rms bunch length), corresponding to the maximum projected emittance growth as shown in Fig. 8(b). CSRTRACK results of bunch length and emittance growth at the end of $\mathrm{BC} 2$, for nominal compression (when $R_{56}=-24.7 \mathrm{~mm}$ ) and for an overcompressed case (when $R_{56}=-29.2 \mathrm{~mm}$ ), are also plotted in Figs. 8(a) and 8(b). We see that they agree with the ELEGANT results.

\section{B. Direct observations of CSR on an OTR screen}

As discussed above, wire scanners instead of OTR screens are used to measure the compressed beam profiles and transverse emittances. All OTR screens after DL1 are compromised by a strong coherent OTR (COTR) signal due to high-frequency longitudinal structure on the electron beam and, hence, these screens cannot be used for accurate beam profile measurements $[9,19,20]$. Nevertheless, CSR signals generated inside a dipole, horizontally separated by the bend from COTR signals, are observed on an OTR screen (OTR21, shown in Fig. 1 as a red dot in the middle of $\mathrm{BC} 2$ ) while adjusting the strength of a horizontal-focusing quadrupole (QM21) a few meters upstream of $\mathrm{BC} 2$. The energy-chirped electron beam entering $\mathrm{BC} 2$ is dispersed horizontally in the middle of $\mathrm{BC} 2$. Thus, the OTR signal from OTR21 emitted by such a beam has a large aspect ratio in the transverse spot size as is shown in Fig. 10(a) (here QM21 = $34 \mathrm{kG}$, the nominal lengthintegrated quadrupole gradient). As we decrease the QM21 strength to $27 \mathrm{kG}$, a CSR signal with an enlarged and more rounded transverse spot appears on the screen [Fig. 10(b)]. At even lower QM21 values, the CSR spot becomes (horizontally) separated from the OTR spot [Figs. 10(c) and 10(d)]. In fact, a part of the CSR signal is clipped off by the OTR screen frame in Fig. 10(d).

Since the OTR camera is sensitive to optical radiation from $350 \mathrm{~nm}$ to about $1 \mu \mathrm{m}$ in wavelength, the observed CSR signal most likely comes from longitudinal density modulation in the beam (microbunching) instead of from the overall bunch shape (the rms bunch length is $70 \mu \mathrm{m}$ in the middle of $\mathrm{BC} 2$ ). The evolution of a microbunched beam in a chicane under the influence of CSR can be described by an integral equation [21,22]. Neglecting the small effect of CSR amplification of microbunching, the amplitude of density modulation in a chicane is coupled to the horizontal emittance by $x-z\left(R_{51}\right)$ and $x^{\prime}-z\left(R_{52}\right)$ trans- 

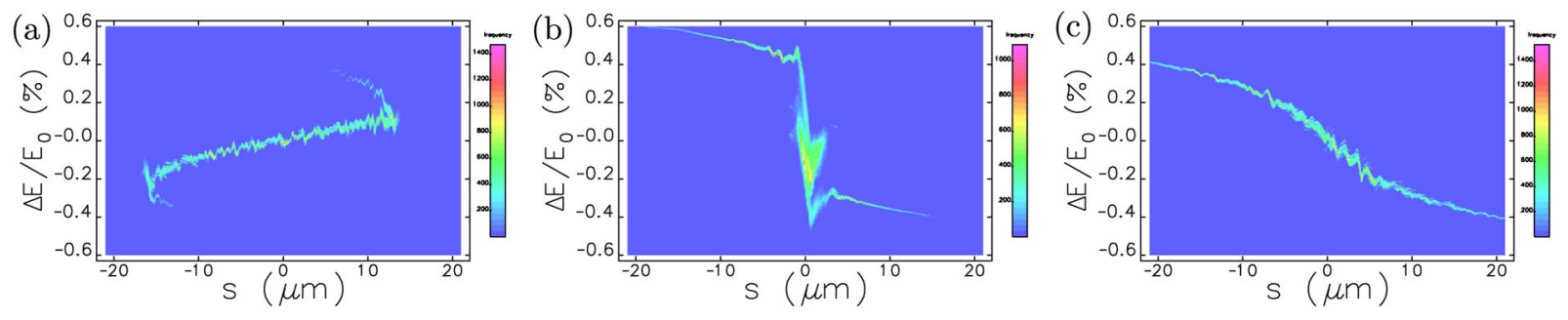

$R_{56}=-24.7 \mathrm{~mm}$

$R_{56}=-27.7 \mathrm{~mm}$

$R_{56}=-29.7 \mathrm{~mm}$
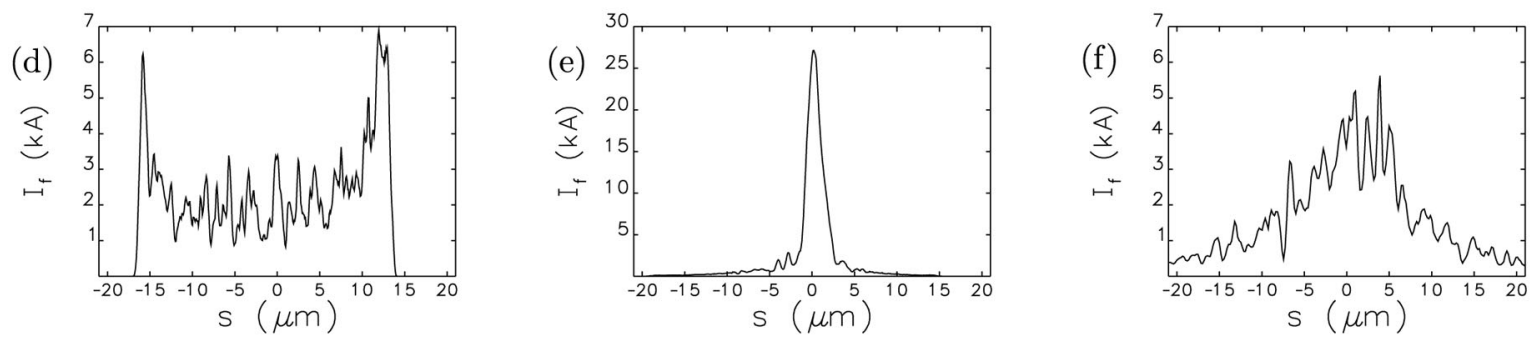

$$
R_{56}=-24.7 \mathrm{~mm}
$$

$$
R_{56}=-27.7 \mathrm{~mm}
$$

$$
R_{56}=-29.7 \mathrm{~mm}
$$

FIG. 9. (Color) ELEGANT simulations of the beam longitudinal phase space distributions (upper plots) and the current profiles (lower plots) in L3 linac at $10 \mathrm{GeV}$ for three $\mathrm{BC} 2 R_{56}$ settings. The bunch head is to the left. (a) $R_{56}=-24.7 \mathrm{~mm}$. (b) $R_{56}=-27.7 \mathrm{~mm}$. (c) $R_{56}=-29.7 \mathrm{~mm}$. (d) $R_{56}=-24.7 \mathrm{~mm}$. (e) $R_{56}=-27.7 \mathrm{~mm}$. (f) $R_{56}=-29.7 \mathrm{~mm}$.
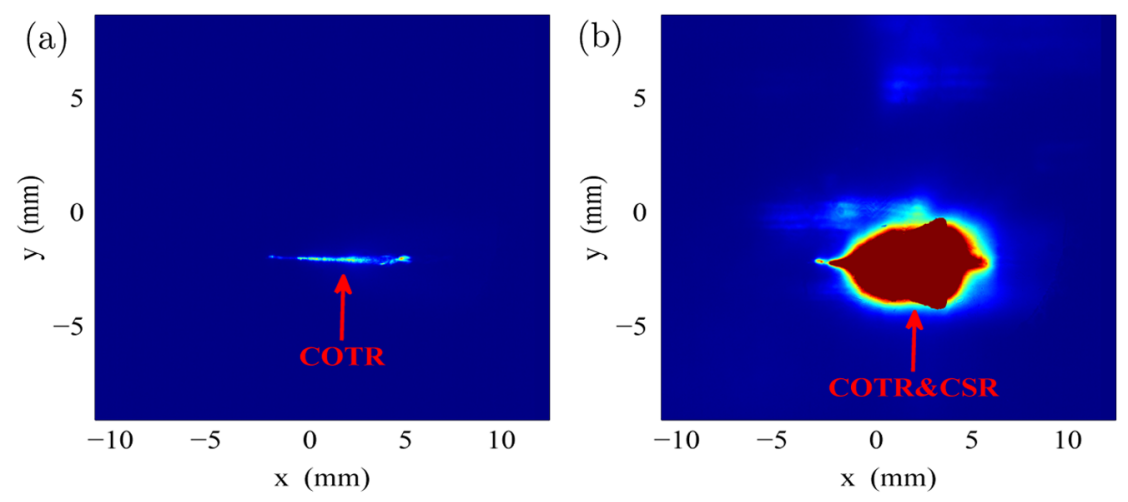

$\mathrm{QM} 21=34 \mathrm{kG}$

$\mathrm{QM} 21=27 \mathrm{kG}$
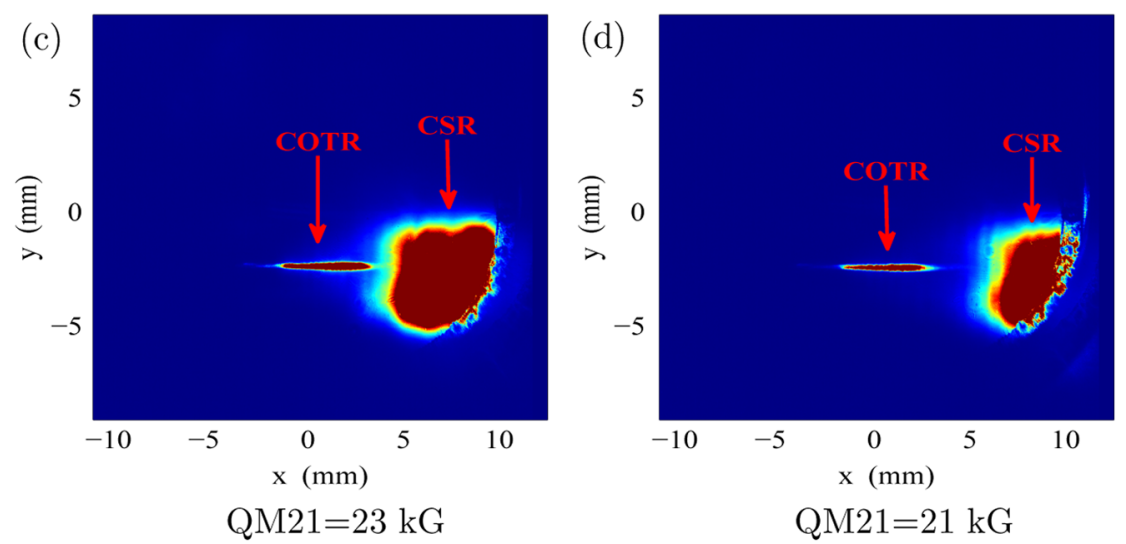

FIG. 10. (Color) Optical radiation pattern observed on an OTR screen upstream of the beginning of the third dipole in $\mathrm{BC} 2$ vs pre-BC2 quadrupole strength (QM21). Both COTR and CSR are detected at this screen location in (b), (c), and (d). (a) QM21 = $34 \mathrm{kG}$. (b) $\mathrm{QM} 21=27 \mathrm{kG}$. (c) QM21 = $23 \mathrm{kG}$. (d) QM21 = $21 \mathrm{kG}$. 
port coefficients [as is shown in Eqs. (26) and (41) of Ref. [22] ]. Let us denote the transverse coupling factor as

$$
\begin{aligned}
C_{x}(k(s) ; s) \propto & \exp \left[-\frac{k^{2}(s) \varepsilon_{x} \beta_{0}}{2}\left(R_{51}(s)-\frac{\alpha_{0}}{\beta_{0}} R_{52}(s)\right)^{2}\right. \\
& \left.-\frac{k^{2}(s) \varepsilon_{x}}{2 \beta_{0}} R_{52}^{2}(s)\right] .
\end{aligned}
$$

Here $k(s)=k_{0} /\left[1+h R_{56}(s)\right]$ is the modulation wave number at distance $s$ from the beginning of $\mathrm{BC} 2$ (the initial point), $k_{0}$ is the initial modulation wave number, $h$ is the initial (relative) energy chirp, and $\alpha_{0}$ and $\beta_{0}$ are the initial horizontal Twiss parameters.

Thus, varying the upstream QM21 strength changes the initial beam divergence [i.e., changes $\alpha_{0}$ in Eq. (2)]. In turn, this affects the microbunching amplitude through the $R_{52}$ from the location of QM21 to the chicane center. If the maximum density modulation is reached inside the second dipole, a strong CSR signal emitted from that location will reach the OTR screen (OTR21), but shifted in the $+x$ direction with respect to the OTR signal generated by the beam. This hypothesis was tested with numerical calculations based on Eq. (2) and the initial Twiss parameters determined by the QM21 setting. We assume an initial modulation wavelength of $1.5 \mu \mathrm{m}$, that is compressed to a wavelength $\lambda \leq 1 \mu \mathrm{m}$ in the second dipole of $\mathrm{BC} 2$, so that optical radiation may be generated and observed by the OTR21 camera downstream. Figure 11 shows the evolution of the transverse coupling factor $C_{x}$ in the second dipole for different QM21 strengths. When QM21 $=34 \mathrm{kG}$ the transverse coupling factor is close to zero so that we do not observe any CSR signal on the screen [Fig. 10(a)]. In this case, the microbunching is washed out by the large nominal horizontal beam divergence at QM21. When QM21 = $27 \mathrm{kG}$ (black solid curve in Fig. 11), the divergence is reduced and the transverse coupling factor reaches a maximum at the end of the second dipole, hence CSR and

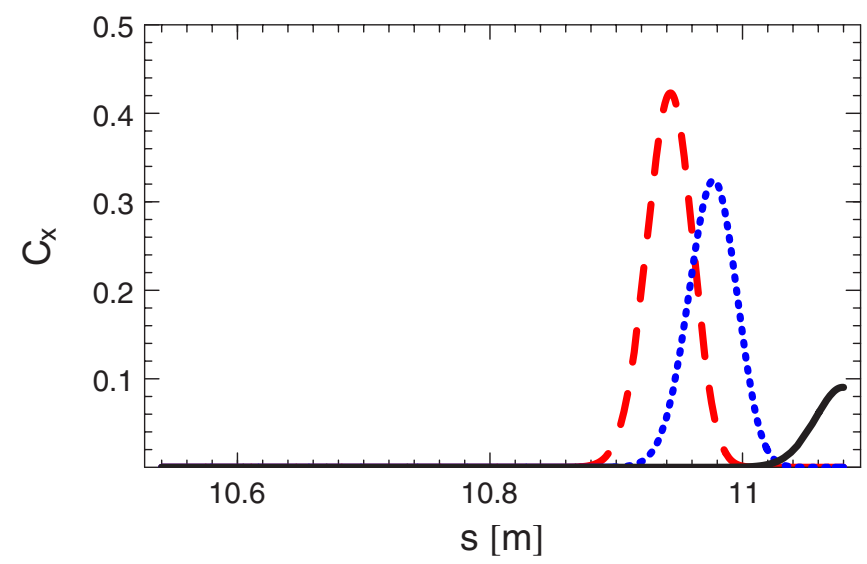

FIG. 11. (Color) Calculated transverse coupling factor in $\mathrm{BC} 2$ second dipole (from $s=10.54 \mathrm{~m}$ to $s=11.08 \mathrm{~m}$ ) for QM21 = $27 \mathrm{kG}$ (black solid curve), $23 \mathrm{kG}$ (blue dotted curve), and $21 \mathrm{kG}$ (red dashed curve). coherent edge radiation are directed to the same horizontal screen location as the beam, as indicated in Fig. 10(b). When QM21 = $23 \mathrm{kG}$ (blue dotted curve in Fig. 11), the transverse coupling factor reaches a maximum at $\sim 10 \mathrm{~cm}$ before the end of the second dipole and strong CSR emitted from this location reaches the screen (which is $0.725 \mathrm{~m}$ downstream of the end of the second dipole), but now shifted horizontally from the beam center by $\sim 5 \mathrm{~mm}$. For $\mathrm{QM} 21=21 \mathrm{kG}$ (red dashed curve in Fig. 11), the transverse coupling factor reaches a maximum at $\sim 13 \mathrm{~cm}$ before the end of the second dipole, and the calculated shift of the CSR center with respect to the beam center is $7 \mathrm{~mm}$. The estimated horizontal shifts of CSR center from beam center agree well with the observed shifts seen in Figs. 10(c) and 10(d). In addition, CSR is emitted with an opening angle on the order of $[\lambda /(2 \pi \rho)]^{1 / 3}$, which explains the much larger vertical CSR spot size seen on the screen $(\sim 2 \mathrm{~mm})$ than the vertical electron beam size.

Finally, we should point out that the microbunching "sharpens up" over a very small distance in $\mathrm{BC} 2$ due to the transverse coupling factor (Fig. 11) and hence will not contribute much to the energy loss and emittance growth in $\mathrm{BC} 2$. This is also indicated in ELEGANT simulations where the slice emittance of the bunch core is unaffected at the nominal BC2 compression [see Fig. 8(b)]. Nevertheless, a microbunched beam can increase the slice energy spread due to the accumulated energy modulation in the linac, which is not measurable by our present diagnostics.

\section{SUMMARY}

We have presented detailed measurements of CSRinduced energy loss and transverse emittance growth in the two LCLS bunch compressors (which are at very different beam energies), and have observed CSR emission induced by a microbunched beam. These measurements and observations are consistent with simulations and analysis. The addition of a Landau-damping laser heater in late 2008 should mitigate beam microbunching and smooth out the high-current spikes after BC2, which may also reduce the horizontal emittance growth there.

\section{ACKNOWLEDGMENTS}

We thank the SLAC engineering, lasers, controls, operations, and rf support groups for their help and dedicated support, and also thank J. Qiang in assisting us using both IMPACT-T and IMPACT-Z codes. This work was supported by Department of Energy Contract No. DE-AC0276 SF00515.

[1] G. Bassi et al., Nucl. Instrum. Methods Phys. Res., Sect. A 557, 189 (2006). 
[2] H. H. Braun et al., Phys. Rev. Lett. 84, 658 (2000); H. H. Braun et al., Phys. Rev. ST Accel. Beams 3, 124402 (2000).

[3] M. Borland and J. Lewellen, in Proceedings of the 2001 Particle Accelerator Conference, Chicago, Illinois (IEEE, Piscataway, NJ, 2001), p. 2823.

[4] P. Emma et al., in Proceedings of the 2003 Particle Accelerator Conference, Portland, OR (IEEE, New York, 2003), p. 3129.

[5] F. Zhou et al., Phys. Rev. ST Accel. Beams 9, 114201 (2006).

[6] B. Beutner et al., FEL2007 Proceedings (2007), p. 18.

[7] M. Borland et al., Nucl. Instrum. Methods Phys. Res., Sect. A 483, 268 (2002).

[8] E. L. Saldin, E. A. Schneidmiller, and M. V. Yurkov, Nucl. Instrum. Methods Phys. Res., Sect. A 483, 516 (2002).

[9] R. Akre et al., Phys. Rev. ST Accel. Beams 11, 030703 (2008).

[10] K. Bane et al., in Proceedings of the 2007 Particle Accelerator Conference, Albuquerque, New Mexico, 2007 (IEEE, Albuquerque, New Mexico, 2007), p. 807.

[11] R. Akre et al., FEL2008 Proceedings, Gyeongju, Korea,
2008.

[12] Z. Huang et al., Phys. Rev. ST Accel. Beams 7, 074401 (2004).

[13] R. Akre et al., Proceedings of the 2001 Particle Accelerator Conference, Chicago, Illinois (Ref. [3]), p. 2353.

[14] J. S. Nodvick and D. S. Saxon, Phys. Rev. 96, 180 (1954).

[15] J. Qiang et al., Phys. Rev. ST Accel. Beams 9, 044204 (2006).

[16] M. Borland, Report No. ANL/APS LS-287, 2000.

[17] M. Dohlus and T. Limberg, FEL2004 Proceedings (2004), p. 18.

[18] J. Qiang et al., J. Comput. Phys. 163, 434 (2000).

[19] J. Frisch et al., 13th Beam Instrumentation Workshop, Lake Tahoe, CA, 2008.

[20] H. Loos et al., FEL2008 Proceedings, Gyeongju, Korea, 2008.

[21] S. Heifets, G. Stupakov, and S. Krinsky, Phys. Rev. ST Accel. Beams 5, 064401 (2002).

[22] Z. Huang and K.-J. Kim, Phys. Rev. ST Accel. Beams 5, 074401 (2002). 